\title{
Recent Medical History - Cataract Surgery
}

\author{
Col R M Youngson
}

MBChB, DO, DTMEH, L/RAMC

\section{Consultant Adviser in Ophthalmology}

Cataract (the name is a semantic curiosity implying a quaint former innocence of anatomy) is, of course, the condition in which the crystalline lens inside the eye becomes opaque. This can happen from physical trauma, such as concussion, or penetrating injury or from a variety of insults ranging from organic poisoning, pre-natal virus infection and hereditary metabolic disease to exposure to the whole spectrum of electromagnetic radiation (a few kilowatts at a few megahertz, if you are near enough) but the enormous majority of cataracts seem to develop simply as a function of age.

New outer lens fibres are continuously produced inside the lens capsule throughout life, and if you live longer than nature (or evolution) intended, this persistent production causes ever-increasing compression and solidity of the centre of the lens, with gradual opacification. In addition to this process, which might be considered almost physiological, actual denaturation and agglutination of the lens fibre protein occurs in the elderly, and nobody really knows the cause. Once the opacities start to form, they usually progress gradually until you can no longer read easily and at that point something has to be done about it. And because of the greatly increased longevity, nowadays, very large numbers of people are turning up at Eye Departments, with cataract. Very few of the aged escape it.

Now there is no pharmacological way of removing opacities from the lens, for these are the result of manifestly irreversible structural changes in the lens substance, so the only remaining resource is to remove the lens altogether. A review of the anatomy of the eye will suggest that this is, on the face of it, a somewhat hazardous adventure, more particularly when you learn that accidental involvement with the vitreous humour (the fluidlike gel lying behind the lens) initiates a succession of unhappy pathological processes which may end in irremediable loss of vision in the eye concerned.

Desperate causes have, however, always engendered desperate remedies, and centuries of evolution and experience have now culminated in a range of techniques by which, without making superhuman demands on surgical expertise, the cataractous lens may be removed with safety and, given that the eye is not the seat of other serious disease, with about a $95 \%$ expectation of restoration of vision.

The first step, of course, is to open the eye, and to do so in such a way as to be able to close it again quickly and accurately. At the same time, the opening must be large enough to permit easy delivery of the lens. For many years the conventional implement was the Graefe knife - a slender, exquisitely sharp-pointed and edged blade, which was passed through one edge of the cornea and out, a full diameter across, at the other - before being drawn upwards with a sawing motion. But the tendency, in recent years, has been towards the easier and safer deliberate and precise placement of the incision by working downwards from above with a knife consisting of a tiny fragment of razorblade or of diamond. One popular method is to cut carefully about half-way through at the corneal margin, pass a suture through the edges of the groove and complete the incision inside the loop. When such a suture is pulled tight, the edges of the wound are perfectly opposed.

The lens is held in place by a delicate "zonule" of fine protein fibres radiating from its periphery to the annular ciliary muscle (which controls its shape in focussing), and before the lens can be removed, this zonule must be broken. Fortunately, there is a fairly linear relationship between age and zonular fragility, so most people with cataracts also have easily ruptured zonules.

Now the last twenty years have seen a quiet revolution in the gentle art of cataract surgery, largely as a result of the almost universal adoption of two entirely new ideas - enzymatic zonulolysis and cryoextraction. To reveal these mysteries, a little background explanation is necessary.

Prior to the introduction of these methods the surgeon had a choice of two main alternatives. Either he could play safe, cut or tear off the front part of the capsule of the lens and squeeze out the contents, leaving behind the rear part of the capsule, or he could attempt to remove the entire lens in its capsule, relying upon the fact that the capsule (which he had, of necessity, to grasp with forceps or some suction device) was usually stronger than the zonule. The trouble with the former procedure is partly that lens contents released into the eye can set up a persistent irritation and partly that the capsule left behind often becomes opaque and has to be opened at a second operation. If much of the front part of the capsule is left, abortive lens fibre growth can continue and lead to trouble. Successful "intracapsular" extraction, on the other 
hand, is followed by a short convalescence and, once the abstracted lens power is replaced by a suitable spectacle correction, full vision.

The immediate object of intracapsular lens extraction is to get the lens out without disturbing the face of the vitreous gel lying behind it. The breakage of the vitreous face and the incarceration of vitreous in the incision is a disaster to which no surgeon can ever become indifferent and it is for this reason that, in spite of its disadvantages, "extracapsular" extraction was so commonly done. Clearly, if the whole capsule is removed, the vitreous face will be exposed and any pressure upon it, or on the outside of the globe is dangerous. So any difficulties in breaking the zonular fibres were liable to make the surgeon's hair stand on end and often led to a catastrophe.

It was in this context that the first notable modern contribution to cataract surgery was made - the introduction by Barraquer, in 1957, of enzymatic zonulolysis.

Chymotrypsin, one of the pancreatic enzymes, present in the pancreatic secretion as chymotrypsinogen, and activated, in the bowel, by enterokinase, rapidly breaks down protein molecules to amino acids at a $\mathrm{pH}$ of 8 or 9. The idea is to inject a solution of the enzyme ( $\alpha$ chyotrypsin is used) behind the iris, wait about three minutes, then wash it out. Of course, the enzyme gets to work on any protein with which it comes in contact, but the mechanical effect on the delicate zonular fibres is far greater than that on any other structure. Often the lens will float free and may easily be slid out. No convincing evidence has been adduced for any important damaging effect on the vitreous or the cornea and there appears to be no practical disadvantage so far as wound healing is concerned. The method has been in almost universal use for over twenty years, during which time many millions of zonules have been digested apparently with almost complete impunity.

Zonulolysis makes possible the intracapsular extraction of lenses in young adults, notwithstanding their tough zonules, but its use is unsafe in children who often have an adhesion between the back of the lens and the vitreous face.

In May 1960, the editor of the British Journal of Ophthalmology received a paper from Tadeusz Krwawicz MD of Lubin, Poland, in which he drew attention to the disadavantages and dangers of the then current methods of grasping the lens, and described a simple device, which he called a cryoextractor. The idea was so simple that one wonders that surgery had to wait so long for it. A metal probe, thermally insulated except for its business end, was cooled well below zero by placing it in a cup of sterile alcohol in a container filled with a mixture of methanol and solid $\mathrm{CO}_{2}$. When it reached the desired temperature, the cryoprobe was wiped 3 dry, dipped in sterile water and brought in contact $\underset{\propto}{\complement}$ with the exposed lens. Rapidly, an iceball would $\Omega$ form which welded, not only the lens capsule, but음 also part of the nucleus, firmly onto the tip of the ? probe, rendering the extraction of the lens, a matter $\vec{F}$ of extreme simplicity, particularly if chymotrypsin $\stackrel{\text { ? }}{9}$ had been used to digest the zonule.

Krwawicz's idea was so obviously sound, (a few $\frac{\bar{c}}{\bar{c}}$ minutes' experimentation with wet fingers in the $\frac{\vec{\sigma}}{\widetilde{D}}$ freezing compartment of a domestic refrigerator will 2 quickly demonstrate the strength of adhesion obtainable) that it was rapidly taken up by other $\overrightarrow{0}$ surgeons. The somewhat crude cooling method des- cribed by Krwawicz has been superseded by others $\vec{\omega}$ and almost every known method of heat abstraction? has been used, in particular adiabatic expansion of $\overline{\vec{J}}$ gases such as $\mathrm{CO}_{2}$ and Freon and the use of liquid? nitrogen. One interesting method is the application $\vec{N}$ of the Peltier effect. Because of the very smallo temperature changes formerly obtainable, this has hitherto been of little more than academic interest, but now semiconductors of low electrical resistan@e and low thermal conductivity (such as bismuth telluride) are being employed, it can give a large $\vec{c}_{\vec{c}}$ temperature gradient, and the equipment can tes

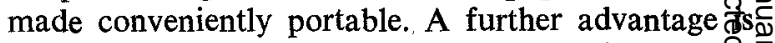
the ease with which temperature control can nofve be exercised (this can be important if, by mischance, $\overrightarrow{0}$ the cryoprobe should freeze on to the wrong tissọe such as the iris or the corneal margin).

When the lens has been slipped out of the the iris (which is often displaced in the process) ? gently reposited and the sutures tied to close the incision. Supplementary sutures are then inserted to $\frac{}{\mathbb{D}}$ reinforce the closure. Modern needles and needle- $\varrho$ holders are of such remarkable quality that fineō suturing is comparatively easy. Almost all surgeons 3 now use operating microscopes or some form of magnifying spectacles or binocular loupe and the use of double operating microscopes, for surgeon and 3 assistant, is becoming increasingly common. These permit a standard of precision hitherto unobtain able but tend to restrict freedom of movement and limit the field of view.

Cryoextraction with zonulolysis has become $a_{0}^{5}$ routine standard technique in cataract surgery and 3 appears to be here to stay. Other recent advancess seem, possibly, of less permanent value. Lens emul-⿳亠丷厂 sification by means of an ultrasonic needle probe, 0 with irrigation aspiration, has had a major vogue? but is now falling into some disrepute. The useo of intraocular lenses to replace extracted cataractous. lenses continues to excite widespread interest buto many patients have suffered unfortunate complica tions and surgeons are becoming more cautious. 